\title{
Analytical approximation for the reflectivity of DH lasers
}

\section{Buus, Jens}

\section{Published in:}

I E E E Journal of Quantum Electronics

Link to article, DOI:

10.1109/JQE.1981.1070735

Publication date:

1981

Document Version

Publisher's PDF, also known as Version of record

Link back to DTU Orbit

\section{Citation (APA):}

Buus, J. (1981). Analytical approximation for the reflectivity of DH lasers. I E E E Journal of Quantum Electronics, 17(12), 2256-2257. https://doi.org/10.1109/JQE.1981.1070735

\section{General rights}

Copyright and moral rights for the publications made accessible in the public portal are retained by the authors and/or other copyright owners and it is a condition of accessing publications that users recognise and abide by the legal requirements associated with these rights.

- Users may download and print one copy of any publication from the public portal for the purpose of private study or research.

- You may not further distribute the material or use it for any profit-making activity or commercial gain

- You may freely distribute the URL identifying the publication in the public portal

If you believe that this document breaches copyright please contact us providing details, and we will remove access to the work immediately and investigate your claim. 
[5] M. Yamanaka, "Optically pumped waveguide lasers," J. Opt. Soc. Amer., vol. 67, pp. 952-958, July 1977.

[6] A. Tanaka, M. Yamanaka, and H. Yoshinaga, "New far-infrared laser lines from $\mathrm{CO}_{2}$-laser-pumped $\mathrm{CH}_{3} \mathrm{OH}$ gas by using a copper waveguide cavity," IEEE J. Quantum Electron, vol. QE-11, pp. 853-854, Oct. 1975.

[7] G. Duxbury and H. Herman, "CW optically pumped far-infrared waveguide laser with variable-output Michelson coupler," J. Phys. E: Sci. Instr., vol. 11, pp. 419-420, 1978.
[8] M. S. Tobin, "Michelson output coupler with 1-dimensional grid for an optically pumped near millimeter laser," in Proc. 4th Int. Conf. Infrared Millimeter Waves Their Applications, FL, IEEE Cat. 79 CH1384-7 MTT, 1979.

[9] F. B. Foote, D. T. Hodges, and H. B. Dyson, "Calibration of power and energy meters for the far infrared/near-millimeter wave spectral region" in Proc. 4th Int. Conf. on Infrared Millimeter Waves Their Applications, FL, IEEE Cat. 79 CH1384-7 MTT, 1979.

\title{
Analytical Approximation for the Reflectivity of DH Lasers
}

\author{
JENS BUUS
}

\begin{abstract}
A simple analytical expression for the reflectivity of the cleaved facets of $\mathbf{D H}$ semiconductor lasers is compared with numerical results, and reasonable agreement is found. The analytical result is conveniently written in terms of waveguide parameters and thus gives a valuable tool for analysis of $\mathrm{DH}$ lasers.
\end{abstract}

$\mathrm{T}$ HE simple plane wave expression for the reflectivity at the boundary between two media with different refractive indexes is frequently used in the analysis of DH lasers. For thin active layers this result is not valid; instead, the reflectivity has been calculated [1] from a numerical solution to the equation obtained from the boundary condition. This paper is often referred to, but due to the complicated equations the results are rarely used.

It turns out that the differences between the simple plane wave result $(\sim 0.32)$ and the numerical result (up to 0.43 for the fundamental TE mode in case of a 10 percent difference in the refractive indexes of the active and passive layers) are quite large, in particular for thin active layers, which are of great practical interest. Hence, a simple and reasonably accurate expression for the reflectivity is highly desirable.

The laser structure is considered as a simple slab with the refractive indexes of the active and passive layers being $n_{1}$ and $n_{2}$, respectively. The thickness of the active layer is $d$ and the polarization is assumed to be parallel to the junction plane (TE mode). This structure was analyzed in a very elegant way by Lewin [2] and an approximate result for the reflectivity was found; unfortunately this work has received very little atten. tion.

The field and power reflection coefficients are denoted $\rho$ and $R$, respectively. For at the plane wave, polarized normal to the plane of incidence, we have in the case of normal incidence

Manuscript received April 20, 1981; revised July 23, 1981.

The author is with the Electromagnetics Institute, Technical University of Denmark, DK-2800 Lyngby, Denmark. on the boundary between a medium with refractive index $n$ and air

$$
\rho=\frac{n-1}{n+1} \text {. }
$$

For the laser structure the effective refractive index is defined by

$$
n_{\text {eff }}=\frac{\beta}{k}=\sqrt{b n_{1}^{2}+(1-b) n_{2}^{2}}, \quad k=\frac{2 \pi}{\lambda}
$$

where the normalized propagation constant for the fundamental TE mode $b$ is the solution to

$$
v \sqrt{1-b}=\arctan \sqrt{\frac{b}{1-b}}
$$

with the usual definition of the normalized frequency $v$, which is also referred to as the normalized thickness

$$
v=k \frac{d}{2} \sqrt{n_{1}^{2}-n_{2}^{2}} .
$$

According to [2] we get for the fundamental TE mode

$$
\rho_{\mathrm{TE}}=\frac{n^{\prime}-1}{n^{\prime}+1}
$$

with

$$
n^{\prime}=n_{\mathrm{eff}} \frac{1+\left(\Delta_{0} / 2 n_{2}^{2}\right)\left(n_{2}-1\right)}{1-\left(\Delta_{0} / 2 n_{2}^{2}\right)\left(n_{2}^{2}-n_{2}+1\right)} .
$$

An expression for $\Delta_{0}$ is given in Appendix II of [2]. It turns out that this expression can also be written in terms of waveguide parameters, introducing the confinement factor $\Gamma$ :

$$
\Delta_{0}=\Delta \epsilon(\Gamma-b) \quad \Delta \epsilon=n_{1}^{2}-n_{2}^{2}
$$

where $\Gamma$ is related to $b$ and $v$ by 


$$
(\Gamma-b)=(1-b) \frac{v \sqrt{b}}{1+v \sqrt{b}}
$$

For a given laser structure $\left(n_{1}, n_{2}, d\right.$, and wavelength $\left.\lambda\right)$ the value of $v$ is found from (4), (3) is solved for $b$, then $\Delta_{0}$ and $n_{\text {eff }}$ can be found using (7), (8) and (2); finally, using (5) and (6) the power reflection coefficient is calculated from

$$
R_{\mathrm{TE}}=\left|\rho_{\mathrm{TE}}\right|^{2} \text {. }
$$

In case of small values of $\Delta_{0}$, i.e., small values of $\Delta e,(6)$ may be further approximated by neglecting a term of the order $\left(\Delta_{0}^{2} / 2\right)$ :

$$
n^{\prime} \simeq n_{\mathrm{eff}}\left(1+\frac{\Delta_{0}}{2}\right)=n_{\mathrm{eff}}\left(1+\frac{\Delta \epsilon}{2}(\Gamma-b)\right) .
$$

For a plane wave incident under a small angle $\theta$ on the boundary between a medium and refractive index $n$ and air it is easily verified that

$$
\rho_{\mathrm{TE}}(\theta) \rho_{\mathrm{TM}}(\theta) \simeq \rho(0)^{2}=\left(\frac{n-1}{n+1}\right)^{2}
$$

where TE and TM denote the respective polarization perpendicular and parallel to the plane of incidence. Assuming this relation to be valid for the slab structure under consideration, the following result is obtained for the fundamental TM mode:

$$
R_{\mathrm{TM}}=\frac{R_{0}^{2}}{R_{\mathrm{TE}}}, R_{0}=\left(\frac{n_{\mathrm{eff}}-1}{n_{\mathrm{eff}}+1}\right)^{2} .
$$

In Fig. 1 the results from the formulas presented in the previous section are compared with the numerical results [1]. It is evident that the analytical expressions are in much better agreement with the numerical results than the plane wave formula. Particularly good agreement is found for active layer thicknesses in the range $0.05-0.2 \mu \mathrm{m}$ where the threshold current density has its minimum value. The agreement is less satisfactory for thickness values around $0.5 \mu \mathrm{m}$, and it is also noted that the extrema occur for lower thickness values than found in the numerical analysis. Results for very low values of the thickness are not shown in [1]. The present analysis shows that the reflectivity for both the TE and TM cases approaches

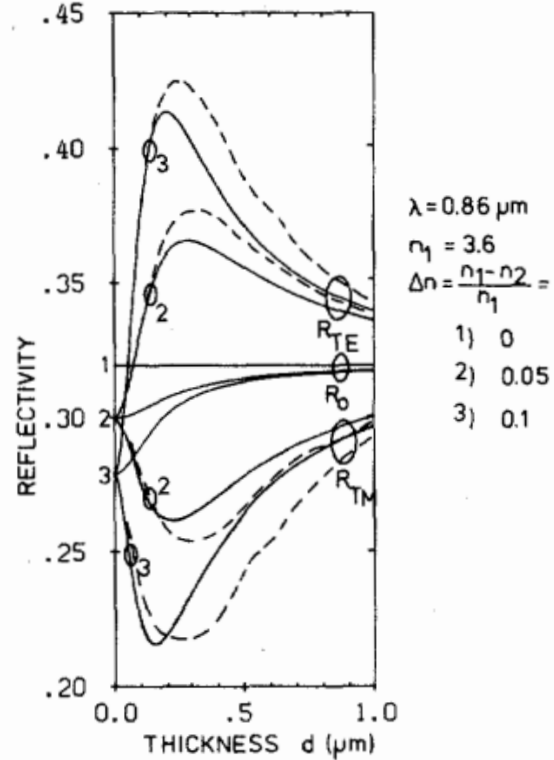

Fig. 1. Reflectivity of the fundamental mode as function of the thickness of the active layer and with the relative refractive index difference as parameter. Case 1 is the plane wave result. The broken curves are reproduced from [1].

the value obtained using $n_{2}$ in the plane wave formula; this is expected since the field in this case resembles a plane wave propagating in the passive layers.

In conclusion, an expression for the reflectivity of $\mathrm{DH}$ semiconductor lasers has been given using normalized waveguide parameters. The calculated results are in satisfactory agreement with results found from a numerical solution of the problem. In particular, a large deviation from the simple plane wave result for active layer thicknesses giving minimum threshold current density is accounted for. The formulas can easily be applied to laser structures with any values of wavelength and refractive indexes, i.e., DH lasers in the GaInAsP system.

\section{REFERENCES}

[1] T. Ikegami, "Reflectivity of mode at facet and oscillation mode in double-heterostructure injection lasers," IEEE J. Quantum Electron., vol. QE-8, pp. 470-476, 1972.

[2] L. Lewin, "A method for calculation of the radiation-pattern and mode-conversion properties of a solid-state heterojunction laser," IEEE Trans. Microwave Theory Tech., vol. MTT-23, pp. 576-585, 1975. 